\title{
Criminologie
}

\section{Les systèmes de justice pénale face à un défi international ?}

\section{Matthew Hall}

Volume 44, numéro 2, automne 2011

Les droits des victimes dans un contexte international

URI : https://id.erudit.org/iderudit/1005790ar

DOI : https://doi.org/10.7202/1005790ar

Aller au sommaire du numéro

Éditeur(s)

Les Presses de l’Université de Montréal

ISSN

0316-0041 (imprimé)

1492-1367 (numérique)

Découvrir la revue

Citer cet article

Hall, M. (2011). Les systèmes de justice pénale face à un défi international ? Criminologie, 44(2), 13-41. https://doi.org/10.7202/1005790ar

\section{Résumé de l'article}

Les droits des victimes de crimes font actuellement l'objet de réformes constantes et d'une attention politique soutenue tant dans la plupart des pays développés que dans les plus hautes sphères des organisations internationales. Le présent article met l'accent sur le caractère international de tels constats en identifiant les normes de services et de soutien que les victimes sont maintenant en droit d'attendre de la part des acteurs de la justice pénale. L'article procédera à une analyse qualitative des déclarations de politique générale qui traitent de la place des victimes en cour et/ou pendant les procédures criminelles et ce, dans neuf juridictions distinctes, et à une analyse détaillée des engagements internationaux fixés par les Nations Unies, le Conseil de l'Europe et l'Union européenne. Les résultats démontrent que les réformes et les attentes en matière de droits des victimes de crimes prennent aujourd'hui la forme d'un processus de mondialisation. Les conséquences d'un tel processus seront explorées à la fois pour les acteurs du système et pour les victimes de crimes elles-mêmes.
Ce document est protégé par la loi sur le droit d'auteur. L'utilisation des services d’Érudit (y compris la reproduction) est assujettie à sa politique d'utilisation que vous pouvez consulter en ligne.

https://apropos.erudit.org/fr/usagers/politique-dutilisation/ 


\title{
Les systèmes de justice pénale face à un défi international?
}

\author{
Matthew Hall \\ Professeur \\ Université de Sheffield, Grande-Bretagne \\ m.p.hall@sheffield.ac.uk
}

\begin{abstract}
RÉSUMÉ - Les droits des victimes de crimes font actuellement l'objet de réformes constantes et d'une attention politique soutenue tant dans la plupart des pays développés que dans les plus hautes sphères des organisations internationales. Le présent article met l'accent sur le caractère international de tels constats en identifiant les normes de services et de soutien que les victimes sont maintenant en droit d'attendre de la part des acteurs de la justice pénale. L'article procédera à une analyse qualitative des déclarations de politique générale qui traitent de la place des victimes en cour et/ ou pendant les procédures criminelles et ce, dans neuf juridictions distinctes, et à une analyse détaillée des engagements internationaux fixés par les Nations Unies, le Conseil de l'Europe et l'Union européenne. Les résultats démontrent que les réformes et les attentes en matière de droits des victimes de crimes prennent aujourd'hui la forme $d^{\prime} u n$ processus de mondialisation. Les conséquences d'un tel processus seront explorées à la fois pour les acteurs du système et pour les victimes de crimes elles-mêmes.
\end{abstract}

MOTS-CLÉS - Victimes, tribunaux, justice pénale, droits des victimes.

\section{Introduction}

Les victimes de crimes, longtemps considérées comme les «acteurs oubliés» (Shapland et al., 1985) du système pénal, ont récemment fait l'objet d'un grand intérêt politique (principes généraux, mais aussi programmes d'action), que cet intérêt émane de juridictions nationales ou d'organisations internationales. Le présent article s'attachera à identifier les normes de services que les victimes sont maintenant en droit d'attendre, via les tribunaux, des acteurs de la justice pénale; en particulier, en ce qui a trait à leur place en cour. L'article procédera à l'anaTraduit de l'anglais par Rabia Mzouji.

Criminologie, vol. 44, $\mathrm{n}^{\circ} 2$ (2011) 
lyse qualitative de neuf juridictions distinctes, incluant une analyse documentaire des législations et des exposés de principe jugés pertinents pour la réflexion menée. Les pays examinés sont les États-Unis, les Pays-Bas, l'Angleterre et le Pays de Galles, l'Écosse, la République d'Irlande, l'Australie, la Nouvelle-Zélande, le Canada et l'Afrique du Sud.

Ces pays ont été sélectionnés pour un certain nombre de raisons. Observés ensemble, et comme expliqués plus bas, ils permettaient d'explorer les diverses avenues empruntées par les réseaux politiques nationaux, transnationaux et internationaux. En pratique, il était nécessaire de choisir des juridictions dans lesquelles les politiques gouvernementales sur les victimes étaient passablement abouties. Premièrement, cela assurait qu'il y ait assez d'information, de rapports et de littérature secondaire à notre disposition pour entreprendre une analyse significative. La plupart des juridictions retenues représentaient ainsi un point de départ fécond pour toute autre juridiction intéressée, elle aussi, à inclure la réforme en matière de victimes dans son agenda politique. Cela étant dit, si la République d'Irlande a par exemple été choisie, c'est essentiellement parce que, quand ce projet a débuté dans les premiers mois de 2008, le Département irlandais de la Justice, de l'Égalité et de la Réforme du droit venait seulement de mettre en place un bureau d'aide aux victimes de crimes, bien loin derrière les dispositions déjà prises par d'autres nations européennes. Il y avait donc encore très peu de littérature sur les réalisations en matière de victimes de crimes dans le système pénal irlandais. Mais cela nous a fourni l'occasion d'examiner la mise en place de telles politiques dans une juridiction et ce, à une étape relativement préliminaire.

Les juridictions retenues ont toutes été marquées par un récent changement significatif quant à la place des victimes dans leurs systèmes pénaux respectifs, notamment sur le plan des mécanismes de soutien, désormais présents et/ou plus conséquents pour les victimes de crimes. On pourrait reprocher à cet échantillon de nations qu'à l'exception des Pays-Bas, toutes les juridictions choisies renvoient à ce que Cavadino et Dignan (2007) appellent des États «néolibéraux». Ces nations peuvent en effet être caractérisées par une orientation politique de droite, un engagement clair pour les principes du libre marché, des taux d'incarcération relativement élevés et des différences de revenus extrêmes. Les Pays-Bas ont été choisis parce qu'on y trouve un centre important pour la recherche et la politique victimologiques, mais aussi et surtout parce que cela permet une comparaison de notre échantillon avec une nation 
«corporatiste conservatrice». En contraste avec les États néolibéraux, Cavadino et Dignan décrivent les pays «corporatistes conservateurs» comme des pays qui, dans le système pénal, mettent l'accent sur la réhabilitation et où il $\mathrm{y}$ a un État social modérément généreux.

Les juridictions choisies ont également été sélectionnées pour représenter aussi largement que possible la divergence des trames historiques et sociales sur lesquelles les réformes de victimes ont été établies. L'Irlande et l'Afrique du Sud ont ainsi connu des histoires récentes de turbulence politique significative comme des soulèvements violents. Au Canada, par exemple, Rock (1990) note que les politiques en matière de victimes faisaient initialement partie d'un programme d'action plus large qui s'appuyait sur une philosophie réparatrice. En Australie, les mécanismes de soutien aux victimes font suite à l'initiative d'organismes particulièrement préoccupés par le soutien aux proches de victimes d'homicides, ainsi qu'aux femmes victimes de viol ou de violence conjugale (David et al., 1990; Rock, 1998, 2007). Tant les autorités canadiennes qu'australiennes ont par ailleurs spécifiquement répondu aux victimisations dont souffrent leurs populations autochtones respectives, avec notamment des avancées dans le champ de la justice restauratrice, en particulier - comme c'est souvent le cas - quand cette justice veille à se présenter et à s'implanter dans un contexte culturel autochtone (Centre des ressources canadiennes pour les victimes de crimes, 2001; Strang, 2001). Les États-Unis, l'Angleterre et le Pays de Galles ont une récente tradition de rétribution dans leurs systèmes pénaux; tradition qui soulève des questions concernant l'existence d'un jeu à somme nulle entre droits des victimes et droits des auteurs des crimes (Hickman, 2004). Cet échantillon de pays permet aussi de dresser des comparaisons entre des juridictions qui, dès la naissance du mouvement des victimes, ont été activement engagées dans des réformes (ÉtatsUnis, Canada, Angleterre et Pays de Galles, Pays-Bas) et un groupe de pays davantage marqués par les avancées les plus récentes de ce mouvement sur le plan international (Afrique du Sud, Australie, NouvelleZélande, Irlande). Le système pénal de l'Écosse est intéressant à étudier parce qu'il partage des affinités à la fois avec l'Angleterre et le Pays de Galles, mais aussi avec le modèle continental. Quant aux Pays-Bas, l'alliance entre l'agenda politique et les chercheurs du champ victimologique est telle qu'on n'en retrouve une trace similaire nulle part ailleurs.

Il faut dès le départ insister sur le fait qu'une recherche comparative entre juridictions est toujours un défi. En particulier quand il travaille 
seul, le chercheur peut ne pas réussir à apprécier les différences subtiles (voire non subtiles) qui existent dans les contextes social, culturel, historique et juridique des pays étudiés. Intégrer neuf juridictions sous une classification relativement uniforme simplifie grandement la démarche d'analyse, mais elle permet en retour de comparer différents types de systèmes. Bien sûr, il ne faut pas minimiser les failles d'une telle entreprise, mais la possibilité de comparaison que permet le projet viendrait quoi qu'il en soit en atténuer les désavantages (par exemple, le fait que le choix limité des pays retenus empêcherait, voire fausserait, toute généralisation sur les décisions prises en la matière par un ensemble plus large et plus diversifié de juridictions).

Étudier ces différents pays se fera à la lumière des engagements internationaux en matière de victimes de crimes tels qu'ils sont établis par les Nations Unies, le Conseil de l'Europe et l'Union européenne. Le but de cet article est de montrer que les réformes entreprises par les systèmes pénaux en la matière connaissent aujourd'hui un processus de normalisation; processus dont les implications seront ici explorées.

\section{Engagements internationaux}

Avant d'examiner les avancées dans les neuf juridictions à l'étude, il est important de prendre connaissance du contexte global des instruments internationaux qui ont en partie conduit à de telles réformes. La Déclaration des Nations Unies sur les principes fondamentaux de justice relatifs aux victimes de la criminalité et aux victimes d'abus de pouvoir de $1985^{1}$ constitue un point de repère important dans cette avancée. Elle exige que les victimes soient traitées avec compassion et dans le respect de leur dignité, qu'elles aient accès à des compensations ainsi qu'à l'information ayant trait au procès criminel, mais aussi que les inconvénients causés par ce procès soient limités au strict minimum. De nombreux pays ont rapidement réagi en mettant en œuvre de telles politiques. La Nouvelle-Zélande y a répondu par la Loi sur les victimes d'infractions criminelles de 1987 (Victims of Offences Act) : les acteurs du système pénal doivent non seulement traiter les victimes avec courtoisie, mais aussi leur permettre d'avoir accès à l'information ainsi qu'à divers services. L'année suivante, le ministère canadien de la Justice a adopté une déclaration de principes visant à uniformiser les droits des victimes. D'autres

1. Que nous appellerons dorénavant dans cet article «la Déclaration de 1985». 
pays, incluant la France, ont mis en œuvre la Déclaration de 1985 dans de nouveaux codes pénaux (Reese, 2000). L'Angleterre et le Pays de Galles n'ont pas directement touché à leur législation, mais ont publié en 1990 une charte des victimes (texte non statutaire) qui promettait aux victimes les traitements évoqués dans la Déclaration de 1985, même si la charte en question manquait de procédures contraignantes (UK Home Office, 1990).

En Europe, le Conseil de l'Europe et l'Union européenne ont institué des mesures centrées sur la victime. La Déclaration des Nations Unies de 1985 a en effet été très vite suivie, la même année, par la Recommandation 85 (11) du Conseil de l'Europe. Cette recommandation cherchait à garder l'esprit de la Déclaration sur le statut à donner à la victime et ce, tant dans la structure organisationnelle de la procédure pénale que dans celle du droit pénal lui-même. Depuis, le Conseil de l'Europe a élaboré une série de conventions et de recommandations sur des questions se rapportant aux victimisations et aux victimes en matière de droit pénal, avec une attention particulière portée aux enfants, aux victimes de la traite des êtres humains (Lee, 2007), ainsi qu'à celles du terrorisme (Pemberton, 2010). Les victimes apparaissaient comme un des enjeux de l'inquiétude manifestée par l'Union européenne dans ses conclusions de Tampere en octobre 1999, conclusions qui élaboraient des plans pour créer un espace de liberté, de sécurité et de justice en Europe. L'Union européenne a depuis lancé nombre de mesures, la plus significative d'entre elles étant sa décision-cadre de 2001 relative au statut des victimes dans les procédures pénales. Document à force exécutoire, cette décision-cadre entend octroyer des droits aux victimes dans toutes les procédures pénales ayant lieu en Europe. Le récent programme de Stockholm sur la Justice et les Affaires intérieures engage de la même façon l'Union européenne à «assurer à toute personne qui devient victime de crimes l'exercice de ses droits ainsi qu'une amélioration des services mis à sa disposition» (para. 2.3.4).

\section{Les droits formels des victimes}

C'est dans ce contexte européen/international que les gouvernements ont réfléchi à la manière d'intégrer les expériences des victimes dans leurs systèmes pénaux respectifs. Cette section met l'accent sur les déclarations-clés relatives aux droits des victimes dans chacune des juridictions retenues; déclarations qui prennent souvent la forme de 
chartes (statutaires ou non) ou de normes de service. Le contenu de tels documents, mais aussi leur force exécutoire, seront discutés. Enfin, la jurisprudence pertinente pour notre propos sera évaluée, qu'elle émane de tribunaux nationaux ou internationaux.

\section{Les chartes des droits des victimes}

La majorité des pays à l'étude se fient à des combinaisons de standards minimaux et d'«attentes légitimes» (JUSTICE, 1998) pour cerner les droits à octroyer aux victimes dans leurs systèmes pénaux respectifs. Les États-Unis font ici exception puisque plusieurs États ont directement incorporé les victimes dans leurs constitutions. La question de savoir jusqu'à quel point de tels amendements produisent des effets pratiques différents de ceux causés par les chartes implantées ailleurs reste toutefois sujette à débat. Dans la plupart des juridictions, ces chartes ${ }^{2}$ sont en effet enchâssées dans le droit: soit parce que leur mise en œuvre est mandatée par la législation (cas du Code déontologique pour les droits des victimes de crimes $^{3}$ ), soit parce que des exemples de meilleures pratiques finissent par prendre la forme de lois (cas de la plupart des chartes australiennes ${ }^{4}$ ).

Parmi les documents les plus pionniers et les plus influents, on retrouve les directives Vaillant qui ont été introduites aux Pays-Bas en 1986 (Wemmers, 1996). Celles-ci ont imposé des obligations à la police et aux avocats de la Couronne afin qu'ils gardent les victimes informées à tous les stades de la procédure pénale, mais aussi qu'ils tiennent compte de l'impact du crime sur les victimes dans le portfolio de l'accusation, voire, si la victime le souhaite, qu'ils engagent une démarche de restitution à l'encontre du condamné. Initialement, les directives Vaillant s'appliquaient uniquement aux victimes de crimes graves, mais en 1987, ces directives ont été élargies et incluaient dorénavant toutes les victimes d'actes criminels. Ces nouvelles règles s'appuient grandement sur la Recommandation 85 (11) du Conseil de l'Europe. Elles se caractérisent par le fait qu'elles mettent l'accent sur une procédure pénale à même de

2. L'article recourt au terme "charte» dans le but de pouvoir décrire les situations diverses mais à contrainte juridique comparable (codes, directives, normes de services) qui émanent des juridictions à l'étude.

3. Domestic Violence, Crime and Victims Act (2004) (Loi sur la violence nationale, le crime et les victimes [2004]).

4. Australian Capital Territory Victims Act (1994) (Loi sur les victimes du Territoire de la capitale australienne [1994]). 
faciliter une démarche restitutive. En 1993, la loi et les directives Terwee ont été reconnues par la législation néerlandaise (Wemmers, 1996). Initialement, ces instruments sont introduits dans trois régions du pays à titre d'expérience à faire évaluer. Deux ans plus tard, en 1995, ils prennent une dimension nationale. Les directives et la loi ont alors reçu une attention significative des médias qui aurait fait en sorte que les autorités ne pouvaient pas les ignorer (Brienen et Hoegen, 2000) ${ }^{5}$. Le constat des deux auteurs est important parce qu'il rappelle que dans les juridictions d'autres pays, les autorités ont dû lutter pour s'assurer que les principes inscrits dans leurs chartes se traduisent en effets pratiques (voir Van Dijk et al., 2008). Dans la plupart des cas, les dispositions de telles chartes n'ont pas valeur de loi et des sanctions légales directes ne peuvent donc pas résulter de leur violation. C'est vrai même là où les chartes s'inscrivent dans des sphères très élevées. C'est le cas de la Charte sud-africaine, qui est censée refléter les droits les plus étendus des citoyens, tels que reconnus par la Constitution nationale. Hanly (2003) a fait des observations similaires en Irlande, où la Charte irlandaise des droits des victimes renvoie à la Déclaration des Nations Unies de 1985 dans son préambule.

C'est clair que la plupart des chartes examinées dans les neuf juridictions à l'étude constituent non seulement une liste de services et/ou de droits pour les victimes, mais aussi une reconnaissance de leur déni traditionnel par les acteurs du système pénal. De tels documents affirment un nouvel engagement pour aborder les victimes avec « empathie, courtoisie et respect» (Irish Department of Justice, Equality and Law Reform, 1999: 6) ou, comme le soutient la Charte australienne:

[Une] victime devrait en tout temps être traitée d'une façon compatissante, constructive et rassurante tant en regard de sa situation personnelle que de ses droits et de sa dignité. (Australian Capital Territory Victims Act, 1994: s. $4[\mathrm{a}])$

Joutsen et Shapland (1989) ont insisté sur l'importance qui a été donnée aux victimes en matière de courtoisie et de respect. Des conclusions similaires ont été constatées tant aux États-Unis (Tyler, 1990; Bazemore, 1998) qu'aux Pays-Bas (Wemmers et al., 1995), mais aussi par l'enquête internationale sur la victimisation criminelle (Van Dijk et al., 2008) et par les enquêtes britanniques sur la satisfaction des témoins (Angle et

5. À la différence des directives Vaillant, inconnues de la plupart des officiers de police (Wemmers, 1996). 
al., 2003). Le droit fondamental d'être traité avec respect par le système pénal constitue donc une des garanties les plus significatives octroyées aux victimes par les diverses juridictions à l'étude. L'enjeu est bien de satisfaire les besoins établis des victimes, et non de répondre à des attentes présumées (voir Rock, 1990).

D'autres droits trouvés dans les chartes de diverses victimes des neuf juridictions peuvent être classés en quatre catégories: la mise à disposition d'information; la protection; la compensation/restitution; et la consultation. Une analyse de contenu des chartes montre que la grande majorité de ces droits implique de garder les victimes informées. Généralement, cette information concerne les avancées de l'enquête, le résultat du procès, les recours en appel, ou encore les motivations qui expliquent les décisions prises par les tribunaux. Un certain nombre de chartes insistent autant sur l'explication qui doit être transmise que sur l'information. Par exemple, les normes nationales écossaises pour les victimes de crimes assurent à celles-ci le droit de recevoir l'information de manière à ce qu'elle soit compréhensible (Scottish Executive, 2005: 3). Les chartes anglaise et irlandaise prévoient aussi que les procureurs rencontrent les victimes, avant la procédure judiciaire, pour répondre à leurs questions. Par ailleurs, les chartes fixent des délais à l'intérieur desquels les victimes doivent être mises au courant des avancées (ou non) de l'enquête. La Charte du Territoire de la capitale australienne exige par exemple une communication mensuelle entre la police et les victimes. En outre, l'Angleterre, le Pays de Galles, l'Écosse, l'Australie Occidentale et la Nouvelle-Zélande détaillent, aux victimes de crimes sérieux qui le souhaitent, toutes les informations auxquelles elles peuvent avoir accès. Généralement, ces informations supposent davantage d'implication des victimes et viennent s'ajouter à celles obtenues d'emblée. Concernant les criminels en détention, les victimes peuvent ainsi avoir de l'information sur leurs auditions de libération conditionnelle, sur leurs libérations provisoires, voire - dans le cas de la NouvelleZélande - sur leur évasion ou leur fuite. On peut cependant noter qu'ailleurs, y compris en Afrique du Sud et en Irlande, toutes les victimes ont droit à cette information.

La Charte irlandaise se distingue des autres chartes pour une raison supplémentaire: elle contient un grand nombre de dispositions qui concernent autant la protection des victimes que l'accès à l'information. La protection inclut le fait: 1) de fournir des services aux témoins vulnérables qui sont amenés à transmettre des preuves à la cour; 
2) d'éviter aux victimes d'entrer en contact avec les accusés; ou encore 3) de leur apporter des services de santé et d'aide psychosociale. La Charte irlandaise va même encore plus dans le détail: 1) un accès garanti à un médecin du même sexe pour les victimes de viol; 2) une politique criminelle qui encourage l'arrestation en cas de violence conjugale ou familiale; 3) des mesures pour protéger les maisons des victimes âgées. Ce souci du détail peut s'expliquer par la priorité que les autorités irlandaises ont voulu accorder ces dernières années aux réponses à apporter au problème de la violence domestique (Bacik, 2002). En comparaison, d'autres chartes donnent des consignes beaucoup plus larges. Pensons par exemple à la Déclaration canadienne des principes fondamentaux de justice relatifs aux victimes de la criminalité ${ }^{6}$ qui soutient que «la sûreté et la sécurité des victimes devraient être considérées à toutes les étapes» (Canada, 2003: Principe 4) ou encore à la Charte sud-africaine qui renvoie à un droit à la protection (p. 8). La Charte néo-zélandaise, elle, se distingue par une absence de mise à disposition de protections spécifiques.

La plupart des chartes contiennent aussi des dispositions concernant la réception, par les victimes, de compensations de l'État et/ou d'une restitution par les criminels ${ }^{7}$. La version de la Charte anglaise est particulièrement détaillée: elle stipule les limites de temps pour la communication des décisions et des appels. Quant à la Charte sud-africaine, elle oblige les tribunaux à imposer des ordonnances de restitution aux contrevenants, comme le fait du reste aussi la Charte irlandaise. La démarche de restitution est aussi mentionnée dans les chartes anglaise et néerlandaise. Dans les cas qui l'exigent, la police est obligée, tant en Angleterre qu'au Pays de Galles, de faire part des données des victimes aux Équipes chargées des jeunes contrevenants (les YOTS - Youth Offending Teams) et ce, en vue de les inclure dans les initiatives de justice restauratrice. Comme déjà mentionné plus haut, les directives Terwee (Pays-Bas) s'étaient particulièrement attachées à mettre en œuvre des mécanismes de restitution (voir Malsch, 1999).

Jusqu'ici, aucun des droits discutés plus haut ne prévoit un quelconque degré d'influence des victimes sur les prises de décision dans les systèmes pénaux, sur ce qu'Ashworth (1998) appellerait les «droits

6. Sur le plan fédéral. Les territoires et les provinces disposent de leurs propres chartes, mais elles sont généralement très proches de l'esprit de la Déclaration.

7. Les exceptions étant la Charte néo-zélandaise et la Déclaration canadienne des principes fondamentaux de justice relatifs aux victimes de la criminalité. 
procéduraux de participation». Néanmoins, la plupart des chartes obligent les acteurs du système pénal à consulter les victimes avant de prendre des décisions spécifiques. Dans certains cas, ces obligations sont formulées de façon très vague. Par exemple, les normes nationales écossaises promettent aux victimes «une consultation en tout temps sur toutes les décisions qui pourraient les affecter» (Scottish Executive, 2005: 4). D'autres chartes disposent de décisions particulières sur lesquelles les victimes peuvent demander des consultations. Tant la charte irlandaise que la hollandaise suggèrent ainsi que les points de vue des victimes devraient être pris en compte dans les décisions en matière de poursuites judiciaires. Le même constat peut être dressé pour l'Angleterre et le Pays de Galles, bien qu'ici les dispositions pertinentes se retrouvent à part, dans le Plaidoyer du procureur (UK Crown Prosecution Service, 2005) ainsi que dans le Code des procureurs de la Couronne (UK Crown Prosecution Service, 2004). De telles attentes sont toutefois tempérées par des clauses qui laissent clairement entendre que les victimes ne peuvent pas dicter les décisions judiciaires:

Le Service des procureurs de la Couronne n'agit pas pour les victimes ou les familles des victimes comme les avocats agissent pour leurs clients. Les avocats de la Couronne agissent au nom du public. (UK Crown Prosecution Service, 2004: paragraphe 5.12)

Parallèlement, les directives de la Nouvelle-Zélande en matière de poursuites judiciaires incluent l'attitude de n'importe quelle victime de crimes comme matière "pouvant être prise en considération" (New Zealand Crown Law Office, 2010 : paragraphe 3.3.2 [m]), mais elles insistent aussitôt sur le fait que la prise en compte de telles attitudes ne sera pas pour autant déterminante. Aux Pays-Bas, les victimes ont le droit de porter plainte auprès de la Cour d'appel quand une accusation n'est pas poursuivie et voient leurs opinions présentées en cour. Bien que la Cour puisse ordonner au procureur de réviser sa décision, en pratique, de telles ordonnances sont rares (Wemmers, 1996).

Plus significativement, et en dépit des appréhensions de certains commentateurs (en particulier Ashworth, 1998 et Sanders et al., 2001), toutes les chartes examinées pour ce projet contiennent des dispositions qui donnent aux victimes la possibilité de faire des déclarations à la cour concernant les répercussions du crime ${ }^{8}$. Ceci dit, seule la Charte

8. Les «Victim Personal Statements» (Déclarations de victimes) en Angleterre, au Pays de Galles et en Écosse. 
irlandaise établit expressément qu'une telle information sera prise en compte par les juges. Quant aux normes écossaises, elles laissent globalement entendre que les points de vue des victimes seront respectés, là où la Déclaration du Canada soutient que:

Les points de vue, les enjeux et les représentations des victimes sont une considération importante dans la procédure pénale. (Canada, 2003: Principe 8)

Pour un certain nombre de juridictions à l'étude 9 , la libération conditionnelle ou provisoire des contrevenants constitue une autre voie d'entrée par laquelle les victimes peuvent demander à être consultées. Une telle consultation est souvent couplée avec des programmes de notification renforcés tel que discuté plus haut.

Une participation consultative aux décisions les plus variées constitue donc une attente légitime dans toutes les chartes des victimes des pays retenus. Néanmoins, l'impact réel de telles consultations reste le plus souvent flou (et ce, qu'elles portent sur la procédure d'inculpation, sur le prononcé de la peine, etc.). Les mêmes observations peuvent être faites pour la plupart des chartes. La plus détaillée d'entre elles est de loin le Code pratique anglais et gallois. Il décrit les attentes de chacune des organisations qui, au sein du système pénal, sont amenées à rencontrer les victimes. Par contraste, la Charte sud-africaine renvoie à quelques organisations spécifiques et décrit les droits des victimes en général et à chaque étape du processus judiciaire.

Une observation-clé des chartes des victimes provenant des neuf juridictions à l'étude concerne la quasi-absence de mécanismes de mise en application qui, comme Jackson (2003) l'argumente, sont pourtant nécessaires pour rendre de tels droits fonctionnels. Aux Pays-Bas, par exemple, le contrôle judiciaire du respect des autorités à l'égard des directives Terwee se limite en fait à une simple procédure d'examen judiciaire (Brienen et Hoegen, 2000). En Angleterre et au Pays de Galles, comme le Plaidoyer du procureur ne donne aucune place aux victimes dans la procédure, celles-ci estiment qu'on ne répond pas à leurs attentes. Sans doute cela impliquerait de se plaindre au Service des procureurs de la Couronne directement. Le Code déontologique anglais pour les victimes de crimes est enchâssé dans la loi, bien que

9. Angleterre et Pays de Galles, Afrique du Sud, Nouvelle-Zélande et Nouvelle-Galles du Sud. 
sa force exécutoire se limite à des procédures de plainte auprès d'organismes de justice spécifiques. Les victimes qui restent insatisfaites du résultat d'une plainte peuvent faire connaître leur mécontentement à leurs parlementaires, qui peuvent à leur tour mentionner le cas à un commissaire parlementaire chargé de l'administration et de l'investigation (UK Home Office, 2005: paragraphe 1.4) ${ }^{10}$. Une nouvelle commissaire pour les victimes et les témoins est chargée de surveiller le suivi des recommandations du Code, bien que des victimes mécontentes ne puissent directement se plaindre à elle. Il est également envisagé que les victimes puissent déposer plainte au Comité consultatif des victimes, mais ni le Comité ni la commissaire n'ont le pouvoir d'investiguer ou de redresser la situation ${ }^{11}$.

Toutes les chartes examinées pour ce projet reposent sur des mécanismes similaires de mise en application qui, en pratique, sont essentiellement limités, on l'a dit, à des procédures de plaintes auprès d'organismes de justice spécifiques. Il est ainsi révélateur que la plupart de ces chartes ne renvoient pas explicitement à des victimes ayant des droits. L'Afrique du Sud constitue à cet égard une exception puisque, tel que noté plus haut, sa charte est présentée en écho à la Constitution nationale. La plupart des chartes témoignent aussi d'une absence d'obligations à remplir par le pouvoir judiciaire, les attentes à son égard se limitant, dans le cas de la Charte irlandaise, à l'exigence de prendre en compte l'impact du crime sur les victimes. La Charte néo-zélandaise exige aussi que les tribunaux prennent en compte le point de vue de la victime quand il y a octroi d'une caution dans les cas de crimes graves.

Dans les juridictions que nous avons retenues, les chartes des victimes constituent les déclarations des droits des victimes les plus facilement accessibles parce qu'elles ont été spécifiquement élaborées pour un public non professionnel. Néanmoins, la quasi-absence de mécanismes de mise en application remet en question le progrès réel fait en ce qui concerne l'introduction effective des droits des victimes. Pour découvrir jusqu'à quel point ces juridictions auraient véritablement changé leur cadre de référence, il importe de regarder de plus près les sources primaires du droit.

10. La Charte néo-zélandaise encourage aussi les victimes à faire part de leurs inquiétudes à un protecteur du citoyen.

11. Le Commissaire parlementaire peut recommander à des organismes de justice de chercher à redresser la situation. 


\section{Les victimes dans la législation nationale}

Parmi les droits octroyés aux victimes, distinguer entre ceux qu'on retrouve dans les chartes et ceux qui apparaissent dans la législation primaire est d'autant plus compliqué qu'il y a une importante superposition des deux dans la plupart des juridictions à l'étude. C'est particulièrement le cas aux Pays-Bas, où les directives, comme les directives Terwee, ont force de loi (Brienen et Hoegen, 2000). La plupart des chartes sont enchâssées dans la législation. Par exemple, la Loi sur les droits des victimes en Nouvelle-Zélande de 2002 (New Zealand Victims' Rights $A c t)$ a adopté la plupart des dispositions telles qu'elles avaient été publiées dans la Charte des victimes de ce même pays, à savoir avec les mêmes larges catégories juridiques (information, protection, compensation/restitution, ou encore participation à des formes de consultation sur l'impact du crime, la procédure d'inculpation, la caution, la libération conditionnelle). La loi donne plus de détails mais pour l'essentiel, elle ne mène pas ces droits plus loin. Le même constat peut être fait pour les mécanismes de mise en application à disposition des victimes, tels que couverts dans la section 49 de la loi. D'ailleurs, la législation dit clairement que le premier moyen à mobiliser pour redresser une situation, ce serait une plainte à «la personne qui, selon les dispositions spécifiques prévues, se doit d'assurer aux victimes la possibilité d'exercer leurs droits» (s. 49 [1] [a]). Si cette première démarche échoue, la victime peut se tourner vers un protecteur du citoyen, l'Autorité indépendante sur les conduites policières ou encore le Commissaire à la vie privée de Nouvelle-Zélande. Néanmoins, là encore, la marge de manœuvre dont bénéficient ces autorités pour aider les victimes lésées n'est pas claire, surtout à la lumière d'une clause de la section 50 (2) qui indique quaucune réparation financière ne peut être ordonnée pour une violation de la section 49. Une disposition similaire a été trouvée dans la $L o i$ anglaise sur les victimes de crime et de violence familiale de 2004 (English Domestic Violence, Crime and Victims Act). Cette loi autorisait la mise sur pied d'un code déontologique pour les victimes de crimes, mais la section 34 (1) de la loi stipule qu'échouer à répondre aux devoirs évoqués par ce code n'engage pas pour autant une responsabilité pénale.

Comme dans le cas des chartes, l'application des droits inscrits dans la législation nationale reste ambiguë dans tous les pays à l'étude. Les chartes de l'État australien et de ses Territoires sont toutes enchâssées dans la législation locale et se fondent sur les principes établis par la 
loi de 1994 sur les victimes de crimes du Territoire de la capitale de l'Australie (ACT), une loi qui, en 1996, sera adoptée par le Comité permanent australien des procureurs généraux. Cette loi de 1994 fournit toutefois peu d'éclaircissements sur la façon dont les droits des victimes peuvent être appliqués, excepté en ce qui concerne la création d'un poste de coordonnateur pour les victimes de crimes. Celui-ci est chargé d'«enquêter sur les pratiques professionnelles et l'administration de la justice pénale chaque fois qu'il a des raisons valables de croire qu'il y a violation des principes de gouvernance édictés par cette loi» (Australian Capital Territory Victims of Crime Act, s. 9 [2]). Le coordonnateur doit alors rapporter les résultats d'une telle investigation au ministre désigné (s. 9 [2]) bien qu'il/elle ne dispose d'aucun pouvoir pour redresser ce type de situation. Ces méthodes visant à renforcer la législation ont été reproduites dans d'autres États et Territoires australiens ${ }^{12}$. La loi victorienne sur la Charte des victimes de 2006 exige simplement la présence d'un organisme qui «informe la victime des procédures à sa disposition pour déposer une plainte» (Victorian Victims' Charter Act, s. 19) et n'offre aucune garantie quant au devenir des plaintes déposées.

En somme, dans la plupart des pays à l'étude, les chartes des victimes n'offrent pas beaucoup plus en termes de droits individuels ou de droits avec force exécutoire. Quand on remonte à leurs lois-cadres, ces chartes contiennent pour l'essentiel des droits sans mécanismes de mise en application. Toutefois, et comme une discussion du cas étatsunien l'illustrera plus loin, les difficultés pour faire appliquer les droits des victimes restent également présentes là où la question des victimes a provoqué un changement constitutionnel.

Les 50 États américains ont édicté des lois détaillant les droits des victimes. Une majorité de ces États ont enchâssé celles-ci dans leurs constitutions respectives à la fin de l'année 2007 (Human Rights Watch, 2008). Le bureau du procureur général de la Californie décrit la mise en application de tels droits dans les termes suivants:

Qu'il s'agisse d'une victime, de l'avocat retenu par la victime, d'un représentant non professionnel de la victime ou encore d'un avocat de la Couronne suite à la requête de la victime, tous peuvent faire appliquer les droits énumérés dans la subdivision (b) de la Section 28 de l'Article 1 de

12. Voir par exemple la Loi sud-australienne sur les victimes de crimes (South Australian Victims of Crime Act, 2001, s. 16 A [2]). 
la Constitution californienne et ce, dans n'importe quel procès, devant n'importe quelle cour d'appel ou toute juridiction qui a une compétence dans l'affaire concernée. La Cour agira promptement face à une telle requête. (Office of the Attorney General of California, 2010: aucune pagination)

Cet extrait laisse entendre qu'obligation est faite aux tribunaux d'assurer aux victimes l'exercice de leurs droits. D'emblée, il importe néanmoins de relever que c'est bien au représentant de la victime et non à la Cour elle-même qu'il revient de soulever l'importance de cet exercice. Le bureau du procureur général de l'Arizona a par contre créé un poste de représentant de la mise en application des droits de la victime, censé adopter des «approches proactives et réactives» (Office of the Attorney General of Arizona, 2009: aucune pagination, italique dans l'original). Les rôles spécifiques de l'agent responsable incluent le fait d'agir comme Protecteur du citoyen pour les victimes de crimes, mais aussi de conduire des enquêtes sur les violations alléguées de leurs droits. Établis en tant qu'amendements constitutionnels, les droits trouvés à la fois en Californie et en Arizona sont renforcés par les cours suprêmes de ces États respectifs. Une telle action n'avait pas encore été prise aux ÉtatsUnis. La Cour suprême du Nouveau-Mexique a publié des ordonnances censées octroyer aux victimes un statut légal pour revendiquer leurs droits ${ }^{13}$, bien que ces ordonnances ne contrôlent pas l'ordre des priorités.

Au palier fédéral, la loi étatsunienne dite Une justice pour tous (Justice for All Act, 2004) traduit une percée majeure selon les partisans d'une forme plus judiciaire des droits des victimes (Doyle, 2008), et ce, du fait qu'elle consacre de tels droits dans le Code pénal étatsunien (18 USC 3771). Cette loi de 2004 réaffirme les droits des victimes à nombre de services: outre la protection, l'information et la compensation, elle donne également aux victimes le droit procédural «d'être raisonnablement entendues dans toutes les procédures judiciaires devant la Cour du district, qu'elles impliquent une libération, un plaidoyer de culpabilité, un prononcé de sentence ou encore une libération conditionnelle» (18 USC 3771 [a] [4]). Plus significativement, cette législation permet aux individus comme au gouvernement fédéral de revendiquer les droits des victimes devant la Cour du district. Si la victime ou le gouvernement ne sont pas encore satisfaits de la mise en

13. Nasci v Pope, Cour suprême du Nouveau-Mexique, 8 novembre 2006, n 29878. 
application de ces droits, ils peuvent déposer une demande auprès de la Cour d'appel pour un bref de mandamus. La poursuite peut également faire valoir qu'une décision de la Cour qui ne respecte pas les droits des victimes constitue un vice de procédure. Dans des circonstances limitées, les victimes peuvent même demander un nouveau procès sur la base du déni de leurs droits (18 USC 3771 [d] [4]); voir Doyle, 2008). Comme elle ne constitue pas un amendement à la Déclaration des Droits des États-Unis, la loi Une justice pour tous ne s'applique pas aux États. Néanmoins, en avril 2008, elle a été incorporée aux règles fédérales qui cadrent les procédures pénales, soit les règles suivies par tous les juges dans les affaires criminelles fédérales. Si cette loi ne permet pas aux victimes de poursuivre le gouvernement fédéral pour violation de leurs droits, elle n'en reste pas moins, au regard des autres juridictions retenues, celle qui assure le plus de mécanismes de mise en application des droits des victimes ${ }^{14}$.

\section{Les droits des victimes devant les tribunaux nationaux}

Dans la plupart des juridictions retenues pour l'étude, le statut qu'occupent les victimes au sein du processus de justice pénale a été remis en question par les tribunaux et ce, à plusieurs occasions. La juridiction d'Afrique du Sud constitue à cet égard une exception. L'enjeu dans ce cas est de mobiliser la Cour suprême ou tout au moins de recevoir une attention judiciaire formelle ailleurs ${ }^{15}$. Le pouvoir judiciaire aux PaysBas, partant d'une expérience en droit civil, est resté lui aussi relativement silencieux sur le sujet, excepté pour confirmer les directives officielles telles que discutées comme source de la loi ${ }^{16}$. Dans d'autres juridictions, les tribunaux ont traité la question des victimes sur un mode fragmentaire. Au Canada, par exemple, la Cour suprême a usé de son influence sur l'élaboration de lois concernant la protection de victimes de viol ${ }^{17}$, notamment en approuvant plusieurs jugements qui

14. Bien qu'un rapport de Human Rights Watch (2008) ait récemment conclu qu'il y avait encore beaucoup à faire concernant la mise en application effective des droits des victimes aux États-Unis.

15. Une information confirmée par la correspondance de représentants du Département sud-africain de la Justice et des Réformes constitutionnelles.

16. Les opinions de la Cour suprême néerlandaise revêtent un caractère non obligatoire pour les cours inférieures même si, en pratique, ses jugements tendent à être suivis (Tak, 2003).

17. Des lois qui visaient à restreindre le contre-interrogatoire des victimes de viol à propos de leur vie et de leurs antécédents sexuels. 
relevaient les conséquences de cette législation sur la possibilité des accusés d'encore bénéficier d'une «défense pleine et entière» telle que promue par la Charte canadienne des droits et libertés fondamentales (s. 11[d]). Dans des affaires antérieures, la Cour avait déjà cherché à restreindre la portée de ces lois $(R \nu$ Seaboyer; $R$. $v$ Gayme [1991: 2 S.C.R. 577]; R. v O'Connor [1995: 4 S.C.R. 411]). Une version révisée des lois concernant la protection de victimes de viol a été jugée nécessaire pour rencontrer les standards constitutionnels dans $R v$ Mills (1999: 3 S.C.R. 668). En posant ce jugement, la Cour a exprimé le besoin de «trouver un équilibre entre droit à la vie privée pour les plaignants et droit à une défense pleine et entière pour les accusés» (L'Heureux-Dubé dans $R$. $v$ Mills, 1999: 672). Que ce soit en Angleterre et au Pays de Galles $(R \nu$ $T ; R v H, 2002$ : Crim. L.R. 73), en Australie ( $M$ [1993, 67 A Crim R 549]), ou encore aux États-Unis (People v MacLeod [Colorado Supreme Court, $\mathrm{N}^{\circ}$ 06SC705. Feb. 4, 2008. Lawyers USA No 9939305]), la jurisprudence se refuse à imposer des règles générales. Elle met plutôt l'accent sur le fait que les tribunaux doivent chercher un équilibre entre les parties, mais aussi pouvoir user de leur compétence quand des preuves qui s'appuient sur la vie et les antécédents sexuels de la plaignante sont présentées (Temkin, 2002).

Le rôle de la victime dans la détermination de la peine a, là aussi, provoqué la constitution d'un corpus de jurisprudence dans la plupart des juridictions retenues, en particulier en ce qui concerne la présentation d'informations aux tribunaux quant aux impacts du crime. En Angleterre et au Pays de Galles, il est ainsi bien établi que les juges chercheraient à cerner l'impact de l'infraction sur les victimes ${ }^{18}$. En comparaison, dans le cas déjà abordé de la Nouvelle-Galles du Sud, la Cour pénale d'appel avait exprimé son insatisfaction à propos de la convention en place dans la plupart des États et Territoires australiens concernant les déclarations de victimes compilées par la police:

[Une] sérieuse faiblesse dans le système actuel tient au fait que ces déclarations de victimes ne sont pas signées ni même reconnues comme appropriées par les victimes concernées et qu'au mieux, elles reflètent l'attitude et l'impression de l'agent de la paix qui les prépare. $(R \nu$ Nicholls $[1991,57$ A Crim R 391, NSW CCA], cité par J. Olsen dans Erez et al., 1996: 209)

18. Attorney General Reference $N^{\circ} 2$ of $1995(R v S)$ [1995] Crim. L.R. 835. Le Code déontologique pour les droits des victimes de crimes renvoie maintenant à un «droit» de la victime à soumettre une déclaration personnelle. 
Les tribunaux étatsuniens ont également insisté sur le droit des victimes de transmettre aux juges les impacts que le crime leur a occasionnés. La Cour suprême étatsunienne a appliqué le même concept d'équilibre à garantir concernant les déclarations de victimes que dans les lois canadiennes sur la protection des victimes de viols (People v MacLeod, $\mathrm{N}^{\circ} 30$, voir plus haut). Dans la première cause type qui a suivi la loi Une justice pour tous, la US Court of Appeals for the Ninth Circuit a décrété qu'un juge de première instance avait mal interprété la loi quand il a refusé aux victimes la permission de décrire à la Cour l'impact qu'avaient eu sur leur vie un blanchiment d'argent et une fraude fiscale ${ }^{19}$. Cette décision suivait un jugement majeur de la Cour suprême dans Payne v Tennessee (501 US, 1991) dans lequel la Cour avait déclaré constitutionnel l'usage de la déclaration de victime dans les cas de peine capitale. Cette position a été récemment confirmée dans les affaires conjointes de Douglas Oliver Kelly v Californie et Samuel Zamudio $v$ Californie (555 US, 2008, № 07-11073 et 07-1142). C'était en l'occurrence les victimes indirectes des meurtres (amis et famille des victimes) qui ont eu le droit de soumettre une déclaration de victime dans les trois cas ci-dessus. Si toutes les juridictions retenues ne sont pas encore prêtes à accepter les déclarations des proches de victimes (par exemple, la Nouvelle-Galles du Sud), il est clair que l'usage de telles déclarations se répand de plus en plus.

Les exemples ci-dessus démontrent que les cours nationales des neuf juridictions à l'étude ${ }^{20}$ ont reconnu la participation des victimes dans le processus de justice pénale. En effet, dans son deuxième cas concernant la loi Une justice pour tous, la Cour suprême note que la législation a été promulguée pour faire des victimes de crimes des acteurs à part entière du système pénal ${ }^{21}$. Ceci étant dit, il est clair que dans la plupart des juridictions retenues, les cours ont résisté aux tentatives des victimes d'exercer un réel pouvoir de décision dans le système pénal. Comme le soutient la Cour suprême irlandaise:

Si poursuivre les contrevenants constitue sans aucun doute un enjeu fondamental dans la lutte pour les droits des victimes de crimes, rendre justice ne doit jamais conduire à privilégier les intérêts des victimes au détriment

19. Kenna v États-Unis. District Court for the Central District of California, 435 F.3d 1011 (9th Cir. 2006).

20. Avec la possible exception de l'Afrique du Sud.

21. États-Unis v Wood, Nos S-08-0195, S-09-0196, 2009 WL 2517175 (Wyo. 19 août 2009). 
de ceux de la Collectivité dans son ensemble. Pour le dire autrement, les intérêts des victimes ne peuvent être satisfaits que s'ils convergent largement avec l'intérêt public (SH v DPP, 2006: 3 IR 575, par C. J. Murray)

De la même façon, dans $R v$ Secretary of State for the Home Department and another, ex parte Bulger (2001 : All E.R. 449), la Cour divisionnaire de l'Angleterre et du Pays de Galles a estimé que la famille de la victime du meurtre ne bénéficiait pas d'un statut justifiant le réexamen d'une décision de justice, y compris concernant la sanction pénale. Dans des instructions pratiques récentes émises par le président de la Haute Cour de justice, la Chambre des lords britannique ${ }^{22}$ a en outre confirmé que les déclarations de victimes ou de proches de victimes ne devaient pas être prises en compte quand leurs opinions portaient sur les «sanctions valables» (2009: WLR [D] 155) ${ }^{23}$. La Haute Cour de Nouvelle-Zélande a suivi la même voie dans $A v$ New Zealand Parole Board and Peter Mana McNamara, en laissant clairement entendre non seulement qu'être une victime ne donne pas de facto le droit de faire une plaidoirie auprès d'un organisme de justice pénale, mais aussi que si plaidoirie il y a, elle n'aura pas nécessairement plus de poids que d'autres considérations pertinentes:

La nécessité, pour la Commission des libérations conditionnelles, d'accorder un «poids équitable» ou juste à ce que la victime dit ne donne pas pour autant à cette dernière la capacité d'étendre la légitimité de ses attentes, simplement en les élevant. Ce «poids juste» doit inclure le concept de pertinence. ( $A \nu$ New Zealand Parole Board and Peter Mana McNamara, HC WN CIV, 2007: 485-2809, paragraphe 17)

Au même titre que l'utilisation de la vie et des antécédents sexuels comme preuve pénale, la plaidoirie de la victime est un critère parmi d'autres à prendre en considération pour la Commission des libérations conditionnelles. L'idée inhérente à cette position est celle de viser un équilibre entre les informations "pertinentes ${ }^{24}$ » provenant des différentes sources. Recourir à l'équilibre rhétorique est devenu un thème récurrent dans les décisions judiciaires de tous les pays à l'étude. On l'avait déjà constaté plus haut avec l'extrait de la Cour suprême cana-

22. Maintenant la Cour suprême.

23. On retrouve un cas analogue avec $R v$ Perks (2000: All ER [D] 763) dans lequel la cour indiquait qu'en certaines circonstances, le choix de la victime de pardonner à un accusé peut devenir un élément pertinent dans la détermination de la peine (Edwards, 2002).

24. La «pertinence» est un terme familier du droit de la preuve pénale dans la plupart des juridictions. 
dienne dans $R v$ Mills. Un principe général, adopté dans la plupart des juridictions à l'étude, semble laisser à la discrétion des juges le soin de déterminer, au cas par cas, le poids à donner à l'information obtenue des victimes. Les cours ont évité, en retour, d'être excessivement prescriptives en de telles matières. Par exemple, dans le cas Angus Sinclair v Her Majesty's Advocate (2007: HCJAC 27), entendu par la Haute Cour de justice écossaise, la Cour a refusé de commenter les arguments de la Couronne laissant entendre que les droits des victimes font partie d'un «procès équitable» (paragraphe 9).

Il est clair que les victimes ont acquis une nouvelle importance dans les décisions judiciaires de la plupart des pays à l'étude, comme il est clair qu'un poids judiciaire s'est ajouté aux droits établis dans les chartes et la législation. Si les droits à la consultation et à la vie privée sont particulièrement importants dans les décisions judiciaires, d'autres droits y sont également présents. Par exemple, la Cour suprême du Canada a établi dans $R$ v R. E. M. (2008: 3 S.C.R. 3, 2008 SCC 51) que pour un juge, fournir à la victime d'un crime des explications sur les raisons d'une condamnation ne doit pas lui apparaître «moins important» que les explications qu'il donnera à l'accusé. Comme discuté plus haut, cet état de fait rappelle simplement le droit qu'a la victime d'être informée et de comprendre les motivations d'une décision judiciaire. En outre, les cas anglais de $R v$ Billam (1986: 8 Cr. App. R. [S] 48) et $R v$ Millberry (2003: 1 Cr. App. R. 25) mettent en évidence que les rabais de peine offerts aux accusés qui plaident coupables avant des procès pour viol reflètent partiellement le fait que les victimes échapperont au stress de devoir fournir des preuves. L'application judiciaire des droits des victimes a donc surtout pour visée de les protéger d'une intimidation et d'un désarroi excessifs. Enfin, il ressort d'un certain nombre de jugements de la Cour suprême irlandaise que, dans cette juridiction, les victimes ont le droit de faire connaître aux autorités compétentes les répercussions que des activités criminelles ont ou auraient sur elles ${ }^{25}$. Ces victimes ne doivent pas pour autant s'attendre à ce que leurs plaintes se traduisent de facto en investigations, en accusations ou en poursuites criminelles, mais si des décisions arbitraires ont conduit à écarter leurs requêtes, ces décisions pourront faire l'objet de révisions par les cours désignées (Fowler v Conroy, 2005: IEHC 26.9).

25. $R \vee$ Dytham Q. B. 722 (un précédent anglais) appliqué par DPP v Bartly Criminal Liability (Dublin 2000: 177). 
Les juges prennent alors note des principes retenus dans les chartes de victimes, les législations associées ou encore les instruments internationaux, et ce, en dépit de l'absence de dispositions qui garantiraient des mécanismes formels de mise en application. Cela implique que la culture et les pratiques professionnelles des juges soient influencées par des mécanismes moins formels. On peut à cet égard se demander si l'argument d'enchâsser les droits des victimes dans un processus de justice pénale ne serait pas mieux soutenu par des changements dans les cultures professionnelles, plutôt que par des changements dans la loi ou par l'entremise d'un précédent jurisprudentiel (voir Hall, 2010).

\section{Les victimes devant les cours internationales}

Nous pouvons maintenant nous intéresser au statut des droits des victimes dans des lois et des jurisprudences internationales qui créent de solides mécanismes de mise en application. C'est particulièrement évident en Europe avec le cas de Maria Pupino (Affaire C-105/03, Maria Pupino), dans lequel la Cour européenne de justice (CEJ) a confirmé qu'une cour nationale devait interpréter la législation interne pour atteindre les résultats poursuivis par la Décision-cadre ${ }^{26}$ de l'Union européenne en 2001 et ce, avant même que des lois nationales aient été adoptées pour formellement mettre en ouvre cet instrument (voir Fletcher et al., 2008). En instituant cette règle, la Cour s'est essentiellement appuyée sur les jugements de la Cour européenne des droits de l'homme (CEDH, voir Arts, 2006: 5). Dans le cas de Katz (Affaire C-404/07, György Katz v István Roland Sós: par. 96), la Cour européenne de justice a statué que les gouvernements nationaux gardaient «un large pouvoir discrétionnaire» concernant les moyens par lesquels ils implantaient les objectifs de la Décision-cadre. Ainsi, même dans des situations où les lois nationales ont légitimement empêché des victimes de fournir des preuves formelles ${ }^{27}$, la Décision-cadre exige que la victime puisse apporter un témoignage susceptible d'être retenu comme preuve $^{28}$.

Pour revenir maintenant à la Cour européenne des droits de l'homme, les cas de Baegen v Pays-Bas (Application No. 16696/90, 26 octobre 1995)

26. Dans ce cas, l'usage de services venant en aide aux enfants constitue une preuve.

27. Par exemple, dans un cas où la victime avait elle-même intenté une poursuite privée.

28. Voir la Commission européenne (2008) pour une discussion autour de la contradiction inhérente à cette position. 
et de Doorson v Pays-Bas confirment qu'assurer l'anonymat aux témoins pour éviter toute intimidation ne viole pas les droits de l'accusé à l'article 6 pour un procès équitable, à condition que les preuves puissent être effectivement contestées. En outre, le cas de Sn v Suède (Application No. 34209/96, 2 juillet 2002) confirme que l'article 6 n'octroie pas à la défense un droit illimité de faire comparaître des témoins en cour (Ellison, 2003). Le cas $S n$ confirme aussi que les témoins peuvent transmettre des preuves par l'entremise des entretiens enregistrés et ce, sans violer l'article 6. Doak (2005) affirme que les victimes pourraient s'appuyer sur les articles 3 et 8 de la Convention si elles s'estiment traitées de façon dégradante ou si l'État échoue à protéger leurs droits à la vie privée quand elles fournissent des preuves. Au même titre que les cours nationales, la CEDH a résisté à des interprétations de la Convention qui accorderaient aux victimes une influence plus explicite sur la détermination de la peine, même si dans $T$ and $V v U K$ (2000: Crim. L.R. 287), les parents de la jeune victime assassinée ont pu présenter leurs observations en cour.

Dans le cadre d'une décision de la Cour internationale de justice, le juge Oda a émis une opinion dissidente qui faisait référence aux droits des victimes dans le cas de LaGrand (Germany $v$ United States of America). Le cas concernait une demande de l'Allemagne à l'État de l'Arizona pour retarder l'exécution de l'un de ses nationaux (Walter LaGrand), condamné à mort pour meurtre. Dans sa critique de l'opinion majoritaire (qui soutiendra l'ordre d'exécution), le juge Oda notait que :

Si les droits de Monsieur Walter LaGrand doivent être respectés sur la base de critères humanistes, la question des droits des victimes de crimes violents (un point qui a souvent été négligé) devrait, de la même manière, être elle aussi prise en considération (Rapport I.C.J. -1 999 [1]: 18, par le juge Oda)

Les droits de participation des victimes ont aussi été reconnus dans le premier procès criminel de la Cour pénale internationale, lors duquel 93 victimes avaient bénéficié du «statut de participant» sous le Statut de Rome (voir Wemmers, 2010). Ces victimes étaient essentiellement des enfants qui avaient été enrôlés comme soldats par l'accusé, Thomas Lubanga, un chef rebelle en République démocratique du Congo. McDermott (2009) a cependant critiqué la manière dont ce procès a été instruit, ce dernier ayant permis des références à des crimes qui n'étaient même pas inclus dans la mise en accusation officielle. 


\section{La «normalisation » des droits des victimes?}

Il est clair que la rhétorique qui entoure les droits des victimes s'est solidement implantée dans les neuf juridictions à l'étude. Généralement, des droits comme ceux qui ont été octroyés aux victimes tendent à se conformer à une liste standardisée de services juridiques. Ils cherchent à traduire les attentes qu'on retrouve dans les instruments juridiques internationaux et incluent les droits à l'information, à la protection, à la compensation et/ou à la restitution, mais aussi des garanties plus générales de traitements compatissants et respectueux de la part des acteurs du système pénal. Autant de droits qui, si l'on en croit les études en la matière, correspondent en fait assez bien aux attentes des victimes elles-mêmes. Les études suggèrent en effet que non seulement les victimes de crimes ne s'attendent généralement pas à exercer un contrôle sur les décisions prises dans le système pénal, mais aussi qu'elles ne le souhaitent tout simplement pas (Shapland et al., 1985). Il est néanmoins clair que les droits de participation des victimes, sous la forme de consultation sur des décisions spécifiques, sont maintenant devenus la norme dans tous ces pays: les points de vue des victimes peuvent même désormais jouer sur les décisions qui font suite à des poursuites judiciaires, sur la détermination de la peine (et en particulier sur les impacts du crime) ou encore sur la libération conditionnelle. Pour autant, si du moins il faut en croire les juridictions retenues, les professionnels de la justice pénale ne se sentent pas contraints par les points de vue des victimes; des points de vue qui constituent tout au plus un des nombreux facteurs pertinents que ces professionnels doivent prendre en compte.

Le fait que des droits similaires ont été découverts dans les neuf juridictions témoigne du caractère mondialisé de l'enjeu de la victime. En Angleterre et au Pays de Galles, le comité JUSTICE a ainsi soutenu que les débats modernes sur les droits des victimes concernent moins le contenu de ces droits que les mécanismes de justice rendue et de responsabilité qui les encadrent (JUSTICE, 1998). Sur ce point, il a été montré que la mise en œuvre des droits des victimes dans ces juridictions tend à être instituée en dehors de la procédure pénale formelle, que ce soit dans des mécanismes de plainte séparés ou avec des protecteurs du citoyen (et leurs équivalents). Des voies qui, dans les deux cas, vont souvent souffrir d'une quasi-absence de pouvoir coercitif et d'une incapacité à rétablir un équilibre rompu. Le manque de dispositions 
exécutoires reste donc criant et pourrait même, selon Jackson (2003), réduire à néant les revendications que les victimes ont maintenant traduites en droits effectifs. Alors que la plupart des pays ont ancré les droits des victimes dans des chartes statutaires, ni les chartes ni leur législation d'application n'offrent de mécanismes de mise en œuvre approfondis. Cela étant dit, l'ancrage de tels droits dans la loi reste une avancée significative par rapport aux listes précédentes de normes de services qui, elles, ne bénéficiaient d'aucun fondement juridique. Comme l'a reconnu le Victim Support Australasia Inc.:

Le Victim Support Australasia Inc. voit dans les obligations prévues par la loi qui sont formulées en «droits» davantage d'autorité, de symbolisme, de reconnaissance et de force exécutoire. Une charte administrative est une forme de quasi-législation donnant corps à des règles qui ne sont pas directement exécutoires et ce, tant dans des procédures civiles que pénales. (Victim Support Australasia Inc., 2009: paragraphe 4.3)

Malgré cet optimisme, l'analyse ci-dessus amène à se demander si, en pratique et dans chacune des juridictions étudiées, cette nouvelle assise statutaire va donner aux chartes une force exécutoire. La réponse tient peut-être dans le fait que l'impact symbolique des droits des victimes sur les pratiques professionnelles judiciaires, mais aussi sur tout le reste de la profession juridique, s'étend maintenant aux cours nationales et internationales.

L'analyse ci-dessus indique que le concept des droits des victimes est maintenant normalisé dans toutes les juridictions retenues pour l'étude: en matière de police comme en matière judiciaire, et dans la pratique juridique en général. Rappelons qu'il n'y a pas si longtemps encore, les droits des victimes étaient au contraire vus comme inhabituels, voire un peu étranges, en comparaison des droits des accusés qui, eux, sont considérés sacrosaints et inhérents au processus même de justice (Ashworth, 2000; Edwards, 2002). Hanly (2003: 8) a critiqué cette tendance à conceptualiser les droits des victimes comme inattendus:

La promotion des droits humains ne devrait pas exiger une justification utilitariste. Nous ne ressentons pas le besoin de justifier la protection des droits des accusés à un procès équitable en référence à certains autres objectifs.

Pour le gouvernement sud-africain, la notion de droits des victimes vient s'enchâsser dans celle, plus large, des droits humains. 
En somme, les effets réels du mouvement pour un ancrage statutaire des droits des victimes peuvent se trouver non pas dans leur judiciarisation croissante mais bien dans la normalisation d'un concept que les cours sont à même d'appliquer. Pour certains avocats des droits des victimes, ce compromis sera insatisfaisant. Leur préoccupation tient en particulier au fait que, compris comme tels, les droits sont condamnés à une application ambiguë, voire incohérente. En effet, un récent rapport de la Commission européenne reproche à la Décision-cadre de l'Union européenne (2001) d'être excessivement vague (Commission des communautés européennes, 2009). Dans une veine similaire, le département sud-africain de la Justice a admis que compte tenu des ambiguiités que contenait la Charte des victimes sud-africaines, ses principes avaient été appliqués «sporadiquement» (South African Department of Justice and Constitutional Development, 2008: 4). À l'opposé, on peut malgré tout noter que les droits des victimes bénéficient maintenant d'une interprétation et d'une application juridiques nourries par les tribunaux tant nationaux qu'internationaux. Quoi qu'il en soit, un argument reste encore monnaie courante: celui qui laisse entendre que pour gagner en légitimité et pour être applicables de façon effective, les droits des victimes devront passer par un changement dans la culture judiciaire bien plus que par la simple implantation de règles juridiques et de mécanismes d'application.

\section{Références}

Angle, H., Malam, S., \& Carey, C. (2003). Witness Satisfaction: Findings from the Witness Satisfaction Survey 2002. Home Office Online Report 19/03. London: Home Office.

Arts, K. (2006). General Introduction: A Child Rights-Based Approach to International Criminal Accountability. In: K. Arts \& V. Popovski (Eds.), International criminal accountability and the rights of children (3-16). Cambridge: Cambridge University Press.

Ashworth, A. (1998). The Criminal Process: An Evaluative Study (2 ${ }^{\text {nd }}$ édition). Oxford: Oxford University Press.

Ashworth, A. (2000). Victims' Rights, Defendants' Rights and Criminal Procedure. In A. Crawford \& J. Goodey (Eds.), Integrating a Victim Perspective Within Criminal Justice: international debates (185-204). Aldershot: Ashgate Dartmouth.

Bacik, I. (2002). Women and the Criminal Justice System. In: P. O'Mahony (Ed.), Criminal Justice in Ireland (134-154). Dublin: Institute of Public Administration. 
Bazemore, G. (1998). The Juvenile Court and the Future Response to Youth Crime: A Vision for Community Juvenile Justice. Juvenile $\mathfrak{G}$ Family Court Journal, 49, 55-87.

Brienen, M., \& Hoegen, H. (2000). Victims of Crime in 22 European Criminal Justice Systems: The Implementation of Recommendation (85) 11 of the Council of Europe on the Position of the Victim in the Framework of Criminal Law and Procedure. Nijmegen: Wolf Legal Productions.

Canada (2003). Canadian Statement of basic Principles ofJustice for Victims of Crime. Ottawa: Ministère de la Justice.

Cavadino, M., \& Dignan, J. (2007). The Penal System: An Introduction (4 $^{\text {th }}$ édition). London: Sage Publications.

Centre des ressources canadiennes pour les victimes (2001). Restorative Justice in Canada. Ottawa: Centre des ressources canadiennes pour les victimes.

Commission des communautés européennes (2009). Report pursuant to Article 18 of the Council Framework Decision of 15 March 2001 on the standing of victims in criminal proceedings. Brussels: Commission des communautés européennes.

Commission européenne (2008). Meeting Report: Experts' Meeting on Victims of Crime Brussels - 17 November 2008. Bruxelles: Commission européenne.

David, J., Stubbs, J., \& Pegrum, F. (1990). Services for Victims of Crime in Australia. Griffith: Criminological Research Council.

Doak, J. (2005). Victims' Rights in Criminal Trials : Prospects for Participation. Journal of Law and Society, 32, 2924-2316.

Doyle, C. (2008). Crime Victims' Rights Act. New York: Nova Publishers.

Edwards, I. (2002). The Place of Victims' Preferences in the Sentencing of "their" Offenders. Criminal Law Review, Septembre, 689-702.

Ellison, L. (2003). Case Note: The Right of Challenge In Sexual Offence Cases: Snv Sweden. International Journal of Evidence and Proof, 7, 1-2.

Erez, E., Leigh, R., \& O'Connell, M. (1996). Victim Impact Statements in South Australia. Canberra: Australian Institute of Criminology.

Fletcher, M., Loof, R., \& Gilmore, B. (2008). EU Criminal Law and Justice. Camberley: Edward Elgar Publishing.

Hall, M. (2010). Victims and Policy Making: A Comparative Perspective. Cullompton: Willan Publishing.

Hanly, C. (2003). Finding Space for Victims' Human Rights in Criminal Justice. Dublin: Irish Law Society.

Hickman, J. (2004). Playing games and cheating: fairness in the criminal justice system. In: E. Cape (Ed.), Reconcilable rights? analysing the tension between victims and defendants (50-64). London: Legal Action Group.

Human Rights Watch (2008). Mixed Results US Policy and International Standards on the Rights and Interests of Victims of Crime. New York: Human Rights Watch.

Irish Department of Justice, Equality and Law Reform (1999). Victims' charter and guide to the criminal justice system. Dublin: Irish Department of Justice, Equality and Law Reform. 
Jackson, J. (2003). Justice for All: Putting Victims at the Heart of Criminal Justice? Journal of Law and Society, 30, 309-26.

Joutsen, M., \& Shapland, J. (1989). Changing victims policy: the United Nations Victim Declaration and recent developments in Europe. In HEUNI (Ed.), The Role of the Victim of Crime in European Criminal Justice System (1-31). Helsinki : HEUNI.

JUSTICE (1998). Victims in Criminal Justice, Report of the JUSTICE Committee on the Role of Victims in Criminal Justice. London: JUSTICE.

Lee, M. (2007). Human Trafficking. Cullompton: Willan Publishing.

Malsch, M. (1999). Victims' Wishes for Compensation: The Immaterial Aspect. Journal of Criminal Justice, 27, 239-247.

McDermott, Y. (2009). The Lubanga Trial at the International Criminal Court. Disponible sur: www.lubangatrial.org/contributors/

New Zealand Crown Law Office (2010). Prosecution Guidelines. Wellington: New Zealand Crown Law Office.

Office of the Attorney General of Arizona (2009). Victims' Rights Enforcement Officer. Disponible sur: www.azag.gov/victims_rights/enforcement.html

Office of the Attorney General of California (2010). Serving Victims. Disponible sur: http://ag.ca.gov/victims.php

Pemberton, A. (2010). Needs of Victims of Terrorism. In R. Letschert, I. Staiger \& A. Pemberton (Eds.), Assisting Victims of Terrorism. Towards a European Standard of Justice (73-142). Houten: Springer.

Reese, C. (2000). The Implementation of the UN Declaration of Basic Principles of Justice for Victims of Crime and Abuse of Power in France. The Victimologist, 4, 1-2.

Rock, P. (1990). Helping Victims of Crime: The Home Office and the Rise of Victim Support in England and Wales. Oxford: Oxford University Press.

Rock, P. (1998). After Homicide: Practical and Political responses to Bereavement. Oxford: Clarendon Press.

Rock, P. (2007). Theoretical perspectives on victimisation. In S. Walklate (Ed.), Handbook of Victims and Victimology (37-61). Cullompton: Willan Publishing.

Sanders, A., Hoyle, C., Morgan, R., \& Cape, E. (2001). Victim Impact Statements: Don't work, Can't work. Criminal Law Review, Jun, 437-458.

Scottish Executive (2005). National Standards for Victims of Crime. Edinburgh: Scottish Executive.

Shapland, J., Willmore, J., \& Duff, P. (1985). Victims and the Criminal Justice System. Aldershot: Gower.

South African Department of Justice and Constitutional Development (2008). Service Charter for Victims of Crime in South Africa. Pretoria: DoJCD.

Strang, H. (2001). Restorative Justice Programs in Australia. Griffith: Australian Criminology Research Council.

Tak, P. (2003). The Dutch criminal justice system: Organization and operation ( $2^{\mathrm{e}}$ édition). The Hague: Netherlands Ministry of Justice. 
Temkin J. (2002). Rape and the legal process ( $2^{\mathrm{e}}$ édition). Oxford: Oxford University Press.

Tyler, T. (1990). Why People Obey the Law. New Haven: Yale University Press.

UK Crown Prosecution Service (2004). The Code for Crown Prosecutors. London: CPS.

UK Crown Prosecution Service (2005). The Prosecutors' Pledge. Disponible sur: www.cps.gov.uk/publications/prosecution/prosecutor_pledge.html

UK Home Office (1990). Victims' Charter: a statement of the rights of victims. London: UK Home Office.

UK Home Office (2005). The Code of Practice for Victims of Crime. London: UK Home Office.

Van Dijk, J., Van Kesteren, J., \& Smit, P. (2008). Criminal Victimisation in International Perspective, Key findings from the 2004-2005 ICVS and EU ICS. The Hague: Boom Legal Publishers.

Victim Support Australasia (2009). A Human Rights Charter for Australia: Submission from Victim support Australasia Inc. Sydney: Victim Support Australasia.

Wemmers, J.-A. (1996). Victims in the Criminal Justice System. Amsterdam : Kugler Publications.

Wemmers, J.-A. (2010). Victims Rights and the International Criminal Court: Perceptions within the Court Regarding the Victim's Right to Participate. Leiden Journal of International Law, 23, 629-643.

Wemmers, J.-A., van der Leeden, R., \& Steensa, H. (1995). What is Procedural Justice: Criteria Used by Dutch Victims to Assess the Fairness of Criminal Justice Procedures. Social Justice Research, 8, 329-350.

ABSTRACT • Victims of crime are now the subjects of intense policy attention and reform across most developed nations, whilst also receiving sustained attention at the highest levels of many international organisations. This paper sets out to emphasise the international character of such developments by identifying common standards of service and support which victims are now told they can expect from criminal justice actors across jurisdictions, with particular emphasis on the place of victims in court during criminal proceedings. The paper draws on a qualitative analysis of relevant policy documents in nine separate jurisdictions as well as detailed analyses of international obligations set down by the United Nations, the Council of Europe and the European Union. The results show that such reforms and expectations are presently undergoing a process of globalisation. The implications of this will be explored both for criminal justice actors in domestic jurisdictions, and for victims of crime themselves.

KEYWORDS - Victims, courts, criminal justice, rights.

RESUMEN - Los derechos de las víctimas de crímenes son en la actualidad objeto de reformas constantes y de una atención política sostenida en la mayor parte de las naciones desarrolladas, así como en los niveles más altos de diversas organizaciones internacionales. El presente artículo destaca el carácter internacional de dichas tendencias mediante la identificación de normas comunes de servicios y apoyo que las 
víctimas pueden esperar actualmente de los diferentes actores de la justicia penal. El estudio se beneficia del análisis cualitativo de declaraciones de política general que tratan del lugar de las víctimas en los tribunales y/o durante los procedimientos penales en nueve diferentes jurisdicciones y del análisis detallado de las obligaciones internacionales establecidas a ese respecto por las Naciones Unidas, el Consejo de Europa y la Unión Europea. Los resultados demuestran que las reformas y expectativas relativas a las víctimas de crimenes se hallan actualmente en proceso de globalización. Las consecuencias de tal proceso se examinan tanto respecto de los actores de la justicia criminal como de las víctimas de crímenes.

PALABRAS Clave - Víctimas, tribunales, justicia penal, derechos de las víctimas. 